time of TB diagnosis. Values at the two time points were compared using Student's paired t-tests.

Results Thirty-one participants were followed up between August 2012 and February 2013. Serum 25(OH)D concentrations were significantly higher post-recovery than at diagnosis (mean 29.7 vs. $12.2 \mathrm{nmol} / \mathrm{L}, \mathrm{p}<0.0001$ ). Participants also had higher mean serum concentrations of $\mathrm{PTH}$, corrected calcium and $24,25(\mathrm{OH})_{2} \mathrm{D}$ post-recovery than at diagnosis $(\mathrm{PTH}, 4.97$ vs. $2.78 \mathrm{pmol} / \mathrm{L}, \mathrm{p}=0.0003$; corrected calcium, 2.50 vs. 2.45 $\mathrm{mmol} / \mathrm{L}, \quad \mathrm{p}=0.03 ; 24,25(\mathrm{OH})_{2} \mathrm{D}, 3.15$ vs. $1.53 \mathrm{nmol} / \mathrm{L}$, $\mathrm{p}=0.004)$. No statistically significant differences in serum concentrations of $1,25(\mathrm{OH})_{2} \mathrm{D}, 4,25(\mathrm{OH})_{2} \mathrm{D}$ or $\mathrm{DBP}$ were seen between the two time points. Differences in serum concentrations of $25(\mathrm{OH}) \mathrm{D}$ at follow-up vs. baseline remained statistically significant after exclusion of 14 participants who were taking supplemental vitamin D at follow-up and / or who had increased their sun exposure since time of diagnosis $(p=0.005)$, and after exclusion of 17 participants whose baseline sample was taken from March to July inclusive ( $\mathrm{p}=0.0003$ ).

Conclusions Vitamin D status of TB patients improved after resolution of tuberculosis. This phenomenon was not explained by differences in vitamin D supplementation, self-reported sun exposure or season of sampling at follow-up vs. baseline. Our findings raise the possibility that vitamin $\mathrm{D}$ deficiency may be a consequence, as well as a cause, of active tuberculosis.

\section{P91 INCORPORATING TUBERCULOSIS STRAIN TYPING DATA INTO ROUTINE CONTACT TRACING INVESTIGATIONS: EXPERIENCE FROM THE FIELD}

${ }^{1} \mathrm{ML}$ Munang, ${ }^{1} \mathrm{C}$ Browne, ${ }^{2} \mathrm{~S}$ Khanom, ${ }^{2} \mathrm{EG}$ Smith, ${ }^{2} \mathrm{JS}$ Evans, ${ }^{2} \mathrm{P}$ Hawkey, ${ }^{1} \mathrm{H}$ Kunst, ${ }^{1} \mathrm{~S}$ Welch, ${ }^{1} \mathrm{ML}$ Dedicoat; ${ }^{1}$ Heart of England NHS Foundation Trust, Birmingham, United Kingdom; ${ }^{2}$ Public Health England Regional Centre for Mycobacteriology, Birmingham, United Kingdom

\subsection{6/thoraxjnl-2013-204457.241}

Strain typing of tuberculosis (TB) isolates by 24 loci mycobacterial interspersed repetitive unit-variable number tandem repeats (MIRU-VNTRs) is now a routine laboratory tool for TB control, but its utility in informing contact tracing and public health action has not been well reported in the United Kingdom. Since November 2011 we have routinely held typing meetings and undertaken cluster investigation. Over 18 months, 68 clusters were discussed. Fifty-five (81\%) clusters were small (2-5 patients), 7 (10\%) were medium (6-14 patients) and 6 (9\%) were large $(>15$ patients, median $=42, \mathrm{IQR}=26-52)$. Enhanced epidemiological investigation was undertaken in $27 / 68$ (40\%) clusters. Typing meetings alone readily identified 20 definite epidemiological links between $46 / 458$ (10\%) cases. In 15 cases, 9 definite or probable links were not supported by genotyping, leading to expanded screening in one workplace. 112 extended interviews were done. A further 23 definite links between $77(17 \%)$ cases, 2 probable links between $5(1 \%)$ cases and 24 possible links between $72(16 \%)$ cases were found. Expanded screening as a direct result of strain typing and cluster investigation occurred in 4/6 settings where non-household transmission was identified (a factory, 2 places of worship, a hospital, a hostel and a pub). An additional 124 contacts were identified. 65 attended screening, 21 latent TB cases were treated and 1 active TB case was found. Routine incorporation of strain typing data in contact tracing improves diagnosis of latent and active infection but requires investment in data management systems and human resource for enhanced epidemiological investigation.

\section{P92 MILIARY TUBERCULOSIS: DATA FROM A MODERN CASE- SERIES IN THE UNITED KINGDOM}

${ }^{1} \mathrm{~N}$ Venkatraman, ${ }^{1} \mathrm{~T}$ King, ${ }^{1} \mathrm{G}$ Woltmann, ${ }^{1} \mathrm{MJ}$ Wiselka, ${ }^{1} \mathrm{D}$ Bell, ${ }^{2} \mathrm{M}$ Pareek; ${ }^{1}$ University Hospitals of Leicester, Leicester, United Kingdom; '2University of Leicester, Leicester, United Kingdom

\subsection{6/thoraxjnl-2013-204457.242}

Background Miliary tuberculosis (mTB), a severe manifestation of TB is classically associated with a high mortality. Modern data on the management and outcomes of mTB in developed world settings are lacking. We reviewed clinico-bacteriological features of mTB cases presenting to a teaching hospital in Leicester, UK serving an ethnically diverse population.

Methods Retrospective descriptive case-series of all notified mTB cases admitted between 2007 and 2012.

Results 41 cases were identified; median age 47 years (IQR:2965 years), $61 \%$ were male and $80.5 \%$ patients were of IndianSubcontinent origin. $92.5 \%$ of patients were foreign-born and median time between UK arrival and diagnosis was 4 years (IQR:1-10 years). 37(90.2\%) patients had an HIV test; 4 were positive (median CD4 count 60;IQR 30-140). Weight loss $(87.2 \%)$ and fevers $(82.9 \%)$ were the most common presenting symptoms. 30 patients $(73.2 \%)$ had abnormal examination findings; predominantly respiratory $(63.3 \%)$. Initial bloods were diagnostically nonspecific apart from lymphopaenia and depressed lymphocyte:monocyte ratio. All patients had radiological evidence of pulmonary miliary nodules. 28/41(68.3\%) patients had neuroimaging: 14/28(50\%) had neuroradiological involvement-predominantly tuberculomas (12/28-42.9\%). Lumbar punctures were undertaken in $73.2 \%$ of patients but only abnormal in 5 patients (17.2\%) (and only 1 with normal imaging). Overall, $16(39 \%)$ patients had evidence of CNS involvement.

32/41(78.1\%) patients were culture positive (all fully-sensitive) with sputum and BAL providing the highest yield. Antituberculous therapy was commenced within a median of 1 day following hospital admission. To date, 30 patients have successfully completed treatment, 3 are still on treatment, 5 have moved away and $3(7.3 \%)$ have died. In those subjects who successfully completed therapy, the lymphocyte:monocyte ratio increased significantly $(\mathrm{p}=0.0201)$. Patients who died had a longer duration between admission and commencing antituberculous treatment (median 8 days; IQR 1-16 days), than those who successfully completed treatment (median 1 day; IQR 0-3 days).

Conclusions In this developed world setting, mTB is not an uncommon presentation. Although there was a high prevalence of co-existing neurological involvement, overall mortality was low. Undertaking diagnostic procedures for culture is important and has a high yield. Early treatment may have resulted in improved outcomes and the lymphocyte:monocyte ratio may help to monitor response to treatment in miliary TB.

\section{P93 SOCIAL RISK FACTORS ASSOCIATED WITH} TUBERCULOSIS IN A HIGH INCIDENCE AREA OF THE UK 
${ }^{1}$ WM Ricketts, ${ }^{2}$ LF Anderson, ${ }^{1}$ VLC White; ${ }^{1}$ Barts Health NHS Trust, London, UK; ${ }^{2}$ Public Health England, London, UK

\subsection{6/thoraxjn-2013-204457.243}

Introduction The London Borough of Newham consistently has the highest incidence of tuberculosis $(\mathrm{TB})$ in the UK $(\approx 120$ / $100,000)$, with case numbers continuing to rise. We sought to investigate social risk factors for $\mathrm{TB}$ as compared with the general population in this borough.

Methods Of 242 adult patients diagnosed with TB in 2012 at the Newham Chest Clinic $170(70.3 \%)$ completed a structured interview assessing for social risk factors. Demographic data was obtained from hospital records. TB case data was compared with 2011 census data using Fisher's exact test or t-tests as appropriate.

Results Data directly comparable to 2011 census data is summarised in Table 1. Cases had a mean of 3.43 (95\% CI 3.07$3.8)$ household contacts, 2.28 (1.41-3.16) non-household family contacts and $2.02(1.71-2.33)$ close friends. Household and family contacts were almost exclusively of the same ethnicity as the

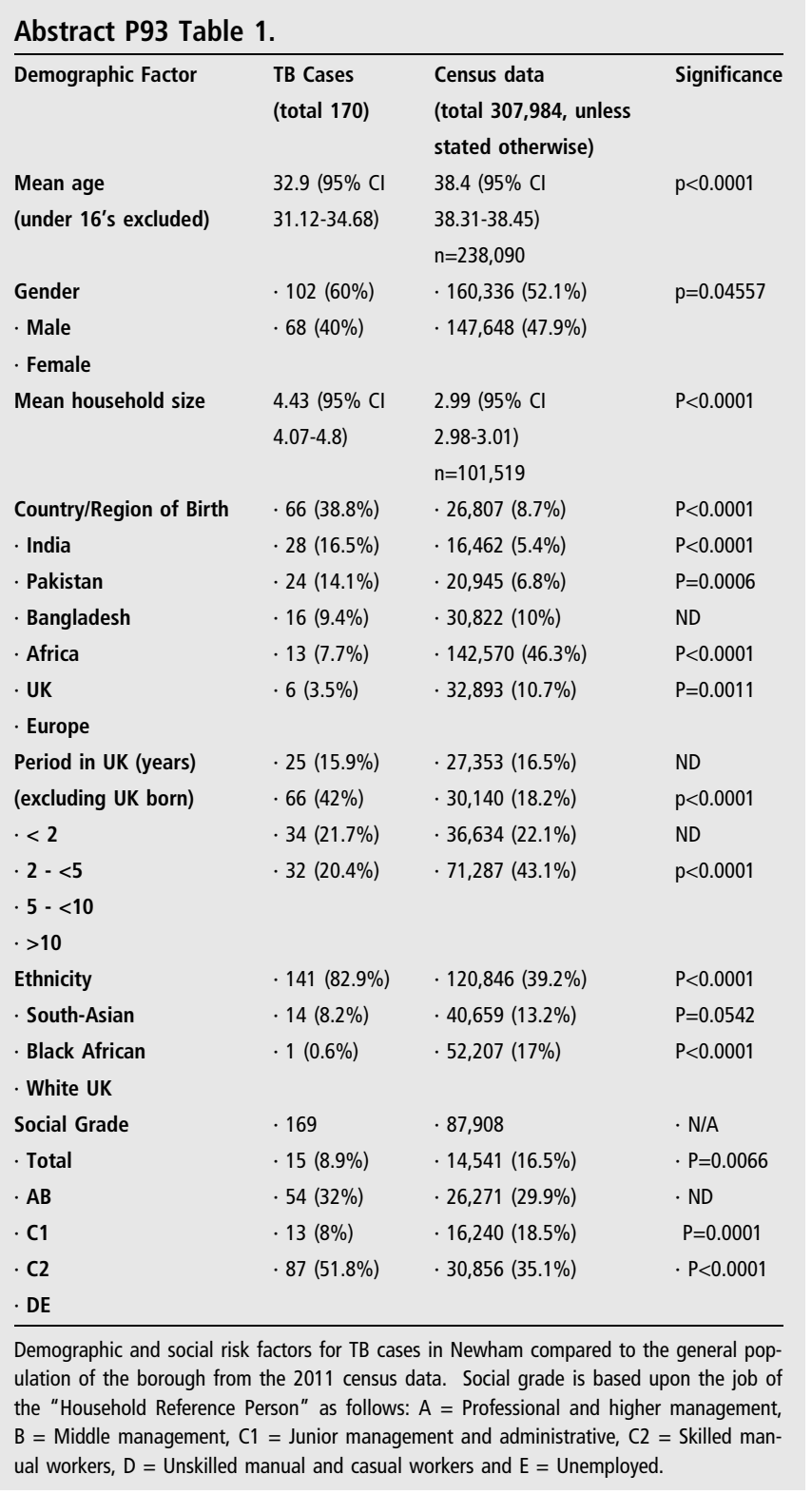

cases, but with $18.4 \%$ of household contacts and $49.2 \%$ of family contacts being UK born, compared with $7.7 \%$ of cases. The majority of friends were of the same ethnicity (86\%) with 18.4\% UK born, $89.4 \%$ of cases had no close friends from a different ethnic group. None of the cohort reported alcohol or drug abuse or had been in prison in the last 5 years, one was currently homeless. Fifteen (8.8\%) reported recent visitors from abroad and 61 (38.9\% of foreign born) reported returning to their country of birth for greater than three weeks, with $75.4 \%$ having returned within the last two years, a further eleven had travelled to a high incidence country in the last ten years, six of whom were UK born travelling to their parents' country of birth. Conclusions In this high incidence area of an otherwise low incidence country TB is significantly more common in those of South-Asian birth and ethnicity. It is more common in young adults who have lived in the UK between two and five years and work in unskilled jobs or are unemployed. This picture is likely driven by $\mathrm{TB}$ reactivation in new immigrants accompanied by a lack of social mixing and suggesting the influence of relative poverty in the development of TB.

\section{P94 RETROSPECTIVE STUDY TO ASSESS THE BACKGROUND INCIDENCE OF HEPATITIS B AND C IN PATIENTS WITH TUBERCULOSIS AND LATENT TUBERCULOSIS AT WHIPPS CROSS HOSPITAL}

VE Beasley, R Anders, M Darmalingam; Whipps Cross University Hospital, London, United Kingdom

\subsection{6/thoraxjn|-2013-204457.244}

Since 2010 our hospital has aimed to test all new patients diagnosed with tuberculosis $(\mathrm{TB})$ or latent $\mathrm{TB}$ for hepatitis $\mathrm{B}$ and C. Guidelines from the NICE recommend all new patients with TB should be tested for HIV. However, no such guidelines exist regarding hepatitis $\mathrm{B}$ and $\mathrm{C}$ screening. We feel patients with undiagnosed hepatitis $\mathrm{B}$ and $\mathrm{C}$ should be identified prior to commencing anti-TB medication due to the potential risk of severe hepatotoxicity. Recently within London, anecdotally two patients with undiagnosed hepatitis treated for $\mathrm{TB}$, subsequently required liver transplantation as a result of significant hepatotoxicity. A retrospective study was conducted to assess hepatitis $\mathrm{B}$ and $\mathrm{C}$ incidence within patients treated for $\mathrm{TB}$ and latent $\mathrm{TB}$ during 2012. Blood results were reviewed for any new hepatitis $B / C$ results. Table 1 shows these results, highlighting that $4.7 \%$ of patients screened in both cohorts tested positive for Hepatitis B or C.

The two additional hospitals within the trust contribute a large number of additional $\mathrm{TB}$ and latent $\mathrm{TB}$ patients, totalling 1115 patients. Through extending the earlier observation of 4.7\% Hepatitis B or C incidence in our hospital, there would be an estimated 51 patients affected across the trust.

Abstract P94 Table 1. Results of TB patients screened positive for hepatitis.

\begin{tabular}{lllll}
\hline & $\begin{array}{l}\text { Total number } \\
\text { of patients } \\
\text { diagnosed } \\
\text { wype }\end{array}$ & $\begin{array}{l}\text { Number of } \\
\text { patients } \\
\text { screened }\end{array}$ & Positive screening result \\
\cline { 4 - 5 } & for hepatitis & Hepatitis B & Hepatitis C \\
\hline Active TB & 153 & 143 & 2 & 6 \\
Latent TB & 59 & 49 & 1 & 0 \\
\hline
\end{tabular}

